\title{
Essais
}

Revue interdisciplinaire d'Humanités

Résister entre les lignes

\section{María Laffitte: La resistencia desde el pensamiento feminista}

Begoña Barrera López

\section{(2) OpenEdition \\ 12 Journals}

Edición electrónica

URL: http://journals.openedition.org/essais/4276

DOI: 10.4000/essais. 4276

ISSN: 2276-0970

Editor

École doctorale Montaigne Humanités

Edición impresa

Fecha de publicación: 15 julio 2016

Paginación: 24-37

ISBN: 978-2-9544269-8-3

ISSN: $2417-4211$

Referencia electrónica

Begoña Barrera López, « María Laffitte: La resistencia desde el pensamiento feminista », Essais [En ligne], 9 | 2016, mis en ligne le 23 octobre 2020, consulté le 31 octobre 2020. URL : http://

journals.openedition.org/essais/4276; DOI : https://doi.org/10.4000/essais.4276 


\title{
María Laffitte: La resistencia desde el pensamiento feminista
}

\author{
Begoña Barrera López
}

María Laffitte (Sevilla, 1902 - Madrid, 1986) desarrolló una amplia trayectoria intelectual como escritora desde la década de los años cuarenta hasta los primeros años ochenta ${ }^{1}$. Su perfil biográfico-intelectual ha de entenderse como la formación progresiva de un pensamiento crítico en torno a tres paradigmas de conocimiento siempre presentes y convergentes tanto en sus obras como en su vida: los avances de la ciencia, la situación sociocultural de la mujer y las artes plásticas modernas. Su modo de articular estas inquietudes y su capacidad para transformarlas en realidades efectivas que le permitieran participar activamente en la vida y en la opinión pública convierten hoy a su figura en un objeto de estudio privilegiado para la investigación sobre el alcance de determinados discursos franquistas y de la resistencia que contra ellos se fue ejerciendo.

Teniendo siempre en cuenta la indisolubilidad de todos los aspectos que ligan y conforman una biografía, este artículo se propone realizar un recorrido que profundice en los aspectos feministas de la trayectoria intelectual de María Laffitte. Si bien ello ha sido abordado de manera más o menos tangencial en obras de diversa temática, en ningún caso se ha tratado la evolución de su pensamiento de manera individualizada, ni se han incorporado a su análisis las ideas que la escritora fue revelando a través de sus textos. En vistas a suplir este vacío, el presente trabajo recoge de manera sintética las progresivas transformaciones del pensamiento y la obra de María Laffitte sobre el tema de la mujer. Para ello, a continuación se propone un recorrido que ilustre el camino que la

1 Laffitte firmó todas sus obras y empleó públicamente su apellido de matrimonio, Campo Alange. En este artículo, así como en otras investigaciones sobre ella, se ha decidido emplear su apellido de soltera respetando la voluntad de la escritora al publicar su primer libro, que quiso firmar con su apellido de familia. Sin embargo, los editores se negaron, por lo que desde entonces continuó firmando con el de matrimonio. Para un análisis en profundidad de la trayectoria de María Laffitte, véase Begoña Barrera, María Laffitte. Una biografía intelectual, Sevilla, Editorial Universidad de Sevilla, 2015. 
escritora transitó desde su primera visión esencialista de la mujer y la maternidad hasta la asunción de las ideas racionalistas e ilustradas, pasando por la realización de uno de los proyectos colectivos más significativos de su biografía, la fundación de un seminario para la investigación sobre la mujer española.

\section{Los primeros pasos de un feminismo esencialista}

Nacida en Sevilla a principios de siglo en una familia aristocrática y conservadora, no fue, por prohibición de sus padres, a la escuela. Tampoco iría a la universidad. Su formación sería durante toda su vida completamente autodidacta. Laffitte definiría el ambiente de su infancia como terriblemente opresor, y esta circunstancia sería algo que tampoco variaría tras su boda. $\mathrm{Su}$ matrimonio con el Conde de Campo Alange supuso el traslado del matrimonio primero a Madrid, y después, durante el gobierno republicano, y dadas las ideas conservadoras de su marido y las suyas en estos primeros años, a París. Volverían a Espańa cuando la guerra civil hubo acabado, y fue entonces, en plenos años cuarenta, ańos duros de represión y de hegemonía del discurso más conservador del franquismo, cuando Laffitte comenzó a desarrollar su labor como escritora.

Años después de haber escrito sus primeras obras, Laffitte aseguraría en su propia autobiografía que la situación social de la mujer había sido siempre una constante en su obra ${ }^{2}$. Si bien ello es cierto y evidente, habría que matizarle en esta consideración que su preocupación transgredió el ámbito de lo social y entró eventualmente en el campo de lo teórico y lo filosófico, pues en cuatro de sus libros (teniendo en cuenta solo los individuales), Laffitte abordó los conceptos de "mujer" y de "feminidad" como piezas claves sobre las cuales cimentar su decidida defensa de la mujer. Sus argumentos necesitaban basarse en un concepto definido de mujer, que en los primeros años vinculará a la maternidad biológica y social (Maria Blanchard, 1944; La secreta guerra de los sexos, 1948), y en las últimas obras le resultará ciertamente problemático de definir, llegando incluso a sugerir la inexistencia de "lo femenino" (La mujer como mito y como ser humano, 1961).

Las primeras reflexiones que Laffitte dejó por escrito surgieron a partir de la "corriente de simpatía” que sintió hacia la obra de la pintora María Blanchard, a la que descubrió durante los años que residió en París. La grata sorpresa que le produjo que quien estuviera tras aquella obra pictórica fuera una mujer propició que la figura de pintora santanderina se convirtiera en el tema de su primer libro, María Blanchard (1944). A mediados de los años cuarenta, y tal y como quedó plasmado en las páginas de esta obra, Laffitte aún tenía una noción bastante débil de la mujer como sujeto, lo que se aprecia en la equi-

2 María Laffitte, Mi atardecer entre dos mundos. Recuerdos y cavilaciones, Madrid, Planeta, 1983, p. 103. 
valencia que estableció entre "ser mujer" y "ser madre". Es decir, entendía la maternidad y la vida familiar como el fundamento de la feminidad. Así, al hablar de la pintora, cuyas deformaciones físicas condicionaron irremediablemente su vida y le ocasionaron importantes trastornos, Laffitte afirmaba que "de no ser por su deformidad su trayectoria en la vida hubiera sido la normal: el amor, un marido, los hijos..."3. Y es que Laffitte tenía un concepto de mujer plenamente arraigado en la función exclusivamente maternal:

Pasiva por una necesidad de su naturaleza, la mujer necesita, sin embargo, la proximidad de la lucha y el trabajo intenso del hombre. Siente así más firme la seguridad del hogar [...] En la vida normal falta a la mujer el motor que da impulso a la idea, porque todas sus energías están fijamente enfocadas en la maternidad [...] El hijo bien formado, sano e inteligente, es, sin duda alguna, la obra de arte con que cada mujer sueña ${ }^{4}$.

En plena posguerra, la maternidad era una representación prefabricada y efectiva que contaba con un objetivo claramente determinado: era un dispositivo orientado hacia la subordinación de las mujeres. De este modo, a través de estas palabras se puede apreciar cómo la definición que Laffitte elabora en 1944 de feminidad y maternidad estaban estrechamente vinculadas con aquellas que se establecían desde el discurso oficial del régimen franquista. De hecho, a pesar de que su concepto de maternidad evolucionaría en cuanto a significado y alcance, durante la década de los cuarenta mantendría un estrecho vínculo con aquel discurso preponderante, ya fuera para asimilarlo acríticamente en 1944, o para resignificarlo estratégicamente en 1948.

Durante los años siguientes a la publicación de su primer libro, Laffitte continuó considerando el problema de la mujer, y sus inquietudes al respecto siguieron aumentando. Aseguraba en sus escritos que "en aquel Madrid de los años cuarenta, el tema de la situación social de la mujer, aunque no era nada nuevo, necesitaba una revisión, y en aquel momento hacerla era especialmente oportuno" . Con todo lo que de deformación retrospectiva y autojustificación puedan tener unas declaraciones escritas más de treinta años después de lo referido, lo cierto es que la determinación que Laffitte tomó sobre esta cuestión en plena posguerra muestran el afán de conocimiento que mantenía la escritora respecto a la situación de la mujer. Apenas tres años después de haberse sumergido en el mundo de las vanguardias artísticas, la escritora pretendía analizar la que ella veía como una "secreta guerra entre los sexos" de carácter universal: "la percibía en el campesino, en el obrero, en el burgués, en el intelectual... Era como una mezcla de actitud protectora, de dominio sexual, de agresividad y de desdén ante toda opinión, aún la más simple, emitida por boca de mujer”6.

3 María Laffitte, Maria Blanchard, Madrid, Hauser y Menet, 1944, p. 26.

4 Ibid., p. 28.

5 María Laffitte, Mi atardecer entre dos mundos, op. cit., p. 64.

6 Ibid., p. 65-68. 
Así, Laffitte acabaría escogiendo "La secreta guerra de los sexos" como título para su segundo libro a partir, según ella, de la lectura de Oswald Spengler, que en La decadencia de Occidente dice así: "He aquí la secreta guerra de los sexos, guerra eterna, que existe desde que hay sexos, guerra silenciosa, amarga, sin cuartel, sin merced. Hay en ella política, batallas, ligas, contratos y traiciones" 7 . Sin embargo, a pesar de mostrarse muy cercana a la lectura de Spengler, Laffitte la tachó de "desconsoladora" y no se mostró conforme con la inmutabilidad que esta interpretación presuponía. Si la cultura había ido elaborándose "con un signo exclusivamente viril, eliminando de ella el vencedor al sexo vencido" , el resultado solo había podido ser el desposeimiento de la mujer de la obra cultural, el enorme desconocimiento que la Historia venía arrastrando de su naturaleza y la distorsión de su "esencia" auténtica: "Su forma psicológica está llena de cicatrices deformativas. Dos mil años de historia han modificado profundamente su ser, y, por último, la asimilación de una cultura de carácter exclusivamente varonil influirá todavía en ella, posiblemente durante siglos, velando su esencia íntima". La deformación y el desconocimiento de lo femenino, de lo sustancial de la mujer, era palpable para Laffitte, por lo que se preguntaba: “¿Pero qué es la feminidad? ¿Debemos ceñirnos con intransigencia rigurosa a ese patrón femenino? ¿No habría que ir hacia una nueva psicología de los sexos?" 10 .

Si en las palabras que ya en 1944 escribía en su obra dedicada a María Blanchard, Laffitte señalaba que "el hijo" era para la mujer la obra de arte más perfecta, el centro de todas sus energías ${ }^{11}$, en 1948, la escritora de $L a$ secreta guerra de los sexos retomó aquella idea de la maternidad pero abordándola desde una perspectiva ampliada:

"Lo femenino" es, sin dudarlo, lo maternal como instinto invencible, con su carga sentimental, con su tara física. El hijo como ambición primordial y, partiendo de ahí, una serie de derivaciones o transformaciones, como es un vivo interés por el ser humano, una sensibilidad especial y perceptibilidad agudizadas, una tendencia a economizar vidas ${ }^{12}$.

En esta obra, Laffitte reclamaba la recuperación de "la verdadera feminidad" que se encontraba "encubierta y casi ignorada", la cual habría "que ir descubriendo poco a poco [para] después rodear de prestigio filosófico y literario la esencia misma de esta feminidad, tan denigrada y desfigurada por la historia". Así, si "la maternidad es la forma primordial y magnífica de la

7 Oswald Spengler, La decadencia de Occidente, Madrid, Espasa Calpe, 1966, p. 296.

8 María Laffitte, La secreta guerra de los sexos, Madrid, Horas y Horas, 2009, p. 145. Primera edición: Madrid, Revista de Occidente, 1948.

9 Ibid., p. 63.

10 Ibid., p. 77-78.

11 María Laffitte, María Blanchard, op. cit., p. 28.

12 María Laffitte, La secreta guerra de los sexos, op. cit., p. 77. 
feminidad", Laffitte defendía que las mujeres habrían de reafirmarse en esta naturaleza, dentro de la que, según la escritora, existían dos tendencias: "La maternidad física, consciente y deseada; [y] la maternidad psíquica, que puede concretarse a los hijos -caso de existir estos- o extenderse, los tenga o no, a la humanidad entera" ${ }^{13}$.

Esta distinción entre maternidad biológica y maternidad psíquica fue fundamental, pues escondía tras de sí dos procesos complementarios que muy sutilmente propiciaban un desplazamiento respecto al concepto central de feminidad como maternidad que el discurso médico-científico y su cobertura ideológica habían promovido. El primer proceso tenía sus raíces en lo que Laffitte había escrito en su libro de 1944, y pasaba por ser una estrategia de esencialización de lo que se pudiera considerar como femenino, esto es, una búsqueda de un núcleo común a la naturaleza de todas las mujeres. Así, como elemento esencializador y diferenciador respecto al otro sexo, Laffitte apostó por la maternidad como instinto. Hasta aquí, su propuesta no habría supuesto una ruptura verdadera con la idea hegemónica de mujer-madre si no fuera porque, como fin de esta búsqueda de una esencia femenina, no estaba el emplearla como mecanismo de opresión o desigualdad, sino como instrumento de dignificación de la mujer y de plataforma desde la que legitimar su actuación más allá del ámbito de lo doméstico. Y efectivamente, ello le llevó a establecer necesariamente una diferenciación entre aquella "maternidad biológica”, la que correspondía a la procreación y el cuidado de los propios hijos (aquella en la que se había apoyado el discurso conservador para recluir a la mujer en el hogar), y la "maternidad psíquica", que poseían todas las mujeres, y que debía servir como plataforma desde la que auparse para poder actuar, como mujer y como madre, en el ámbito de lo público. Esta doble naturaleza de lo maternal era la que había que batallarle a la opresión de "lo viril" para que pudiera ser dignificada y ensalzada, permitiendo con ello que su influencia se extendiera más allá del hogar.

Esta estrategia contó, además, con el respaldo firme que Laffitte supo buscarse en la tradición ilustrada de defensa de los derechos de la mujer que ella tanto admiraba y ponderaba. Así, la huella del pensamiento liberal de Concepción Arenal (1820-1893) en cuantas obras escribiera Laffitte fue la mejor evidencia de lo que Joan W. Scott ha definido como "eco de la fantasía", esto es, la necesidad de establecer una genealogía y de recuperar un legado mediante el que identificarse retrospectivamente con los actores de un pasado histórico feminista. ${ }^{14}$ Ciertamente, la admiración por la escritora del siglo XIX fue una constante en la obra de Laffitte desde la década de los cuarenta. Y no

13 Ibid., p. 86.

14 Véase Joan Wallach Scott, "El eco de la fantasía: la historia y la construcción de la identidad", Ayer, ${ }^{\circ} 62$, 2006, p. 11-138. 
solo porque en La secreta guerra de los sexos se explayara en alabanzas a la que entendía como "la más honesta y exquisita feminidad de su siglo" sino porque, aunque sin hacerlo explícito, Laffitte recogía buena parte de su pensamiento feminista y de su defensa de la maternidad social como dignificación y como función de la mujer.

Como ha indicado Nerea Aresti, Concepción Arenal, en una estrategia muy semejante a la que un siglo después llevaría a cabo María Laffitte, se centró en la búsqueda y asignación de unos valores diferentes, específi$\cos$ y comunes a las mujeres. En esta búsqueda, que formaba parte de un proyecto de su enaltecimiento moral de la mujer, la maternidad se convirtió en el centro de su argumentación, "una maternidad que pasaba de ser una condición posible en la vida de las mujeres, un estado que debía competir con otros igualmente legítimos y deseables, a adherirse a la feminidad como una naturaleza que definía la existencia de toda mujer" ${ }^{15}$. La consubstancialidad de lo femenino y lo maternal, y la urgencia de que este factor fuera aprovechado para la mejora social conformaban la propuesta de Arenal: la maternidad social, un sentimiento, predisposición y capacitación que ha de extender su acción directa fuera del hogar doméstico. Estas tareas, que se entendían como aquellas asociadas a la ayuda, el cuidado, la protección, la beneficencia y la educación eran la prolongación natural de la maternidad. Teniendo en cuenta la trayectoria de Concepción Arenal, resulta lógico que cuando Laffitte quiera referirse a su labor la describiera como "desbordante fuerza maternal" 16 .

El paralelo que se estableció entre los proyectos de las dos escritoras tiene su proyección en la defensa de un mismo tipo de estrategia feminista. Como se ha visto, el propósito emancipador que ambas persiguieron se sustentaba en la construcción de una identidad colectiva de las mujeres cuyo núcleo era la maternidad. Era, por tanto, una esencia construida, creada a partir de la acuñación de conceptos y discursos que perseguían la reubicación de las mujeres en la sociedad y en la cultura. Hasta aquí, en cuanto a construcción del lenguaje, esta estrategia sería la misma que la del discurso médico oficialista promulgado por el régimen, que por su parte también construyó un relato de lo que "auténticamente era" (y por tanto "debía ser") la mujer. Lo que lo diferenciaba de él, entonces, no era tanto la defensa de una naturaleza diferencial en la mujer, sino el aprovechamiento que autoras como Arenal y Laffitte hicieron de esta naturaleza, convirtiéndolo en elemento identitario y, en último término, enaltecedor y dignificador de las mujeres. Esta estrategia feminista sería, en tanto que esencializadora de la identidad femenina, el núcleo de la teorización del conocido como "feminismo de la diferencia", que, en base a la supuesta existencia de una identidad compartida, profundizaría aún más en las diferencias entre los dos géneros establecidos.

15 Nerea Aresti, "Juegos de integración y resistencia. Discursos normativos y estrategias feministas (1860-1900)", Historia Social, no68, 2010, p. 25-46, p. 33.

16 María Laffitte, La secreta guerra de los sexos, op. cit., p. 65. 
El interés de Laffitte por Concepción Arenal le llevó, en los años siguientes, a ahondar en su figura y en la de sus contemporáneas en varios escritos. En 1964 publicaría por encargo de la editorial Aguilar un libro titulado La mujer en España. Cien años de su historia, donde señalaba que las obras de Arenal "continúan teniendo un interés vivo y son fuente del más puro sentido común. A pesar de todo -añadía- hoy son casi desconocidas para sus compatriotas" ${ }^{17}$. En esta línea, su interés por Concepción Arenal se plasmó en otras dos publicaciones de la década de los setenta que venían a corroborar la sintonía que la escritora sentía no solo con ella, sino con otras tantas escritoras liberales decimonónicas. En 1973, la escritora plasmó en una obra titulada Concepción Arenal. 1820-1893. Estudio biográfico documental la concienzuda investigación que había realizado sobre su figura ${ }^{18}$. Poco después, en 1977, Laffitte prologó las memorias de la Condesa de Espoz y Mina con un texto en el que sintetizaba los rasgos que para ella eran más ponderables de la escritora: sus preocupaciones sociales, su pensamiento liberal y el vínculo comunitario que unió a la de Espoz y Mina con Concepción Arenal, Rosalía de Castro y Emilia Pardo Bazán ${ }^{19}$.

\section{Hacia el feminismo ilustrado}

En 1950 y 1958 La secreta guerra de los sexos fue reeditada en Revista de Occidente. El prólogo que acompaña a la segunda edición ha sido traído a colación por algunos estudios como testimonio fundamental para rastrear la repercusión en España a finales de los años cuarenta de una de las obras fundacionales del feminismo, Le Deuxième Sexe de Simone de Beauvoir ${ }^{20}$. Efectivamente, en 1949, un año después de la aparición del libro de Laffitte, se publicó en Francia el libro en dos tomos de Beauvoir, del que la primera no pudo dejar de hacerse eco, recalcando, no obstante, que este había aparecido un año después de su obra. Aunque Laffitte llegara a reconocer su interés por el concepto de "Otredad" acuñado por Beauvoir, fue el concepto de raíz ilustrada de "androcentrismo" (que Laffitte no empleará con este nombre, pero que subyacerá en los escritos de los cincuenta) y la propuesta de Beauvoir de desechar la existencia de la mujer como inmutabilidad lo que verdaderamente caló hondo en los planteamientos de la escritora. Porque si a Laffitte le interesaba profundizar en las tesis de Beauvoir y corroborarlas con las ideas cientí-

17 María Laffitte, La mujer en España. Cien años de su historia, Madrid, Aguilar, 1964, p. 105.

18 María Laffitte, Concepción Arenal (1820-1893). Estudio biográfico documental, Revista de Occidente, 1973.

19 María Laffitte, "La Condesa de Mina: una línea bien trazada", prólogo a Juana Espoz y Mina, Condesa de Espoz y Mina. Memorias, Madrid, Ediciones Giner, 1977, p. 7-13.

20 Gloria Nielfa Cristobal, "La difusión en Espańa de El Segundo Sexo de Simone de Beauvoir", Arenal, 9, nº1, 2002, p. 151-162. 
ficas, era porque percibía un fenómeno contemporáneo y cercano a ella que necesitaba explicar, y aquella diferenciación entre lo biológico y lo construido social y culturalmente le ofrecía el marco de análisis desde el que hacerlo.

En 1961, Laffitte publicaba una recopilación de breves ensayos titulado $\mathrm{La}$ mujer como mito y como ser humano en la que la escritora afirmaba que "el ideal de mujer sumisa, dulce y analfabeta", propio de un ser "desposeído de cultura", estaba desapareciendo. Y de ello derivaba lógicamente la siguiente pregunta: "¿ Cómo explicarnos, por otra parte, que al desaparecer algo que consideramos como consubstancial con la mujer, la mujer misma, el ser humano femenino, permanezca inconmovible ?"21. Ya desde su libro de 1948, Laffitte abordaba cuestiones de este tipo, señalando siempre el trance en el que vivía la mujer de su tiempo. Sin embargo, después de leer a Beauvoir el asunto aparece mucho más claramente planteado. Laffitte entendía que no era la "mujer" la que estaba desapareciendo, era el modelo histórico y cultural lo que se estaba disolviendo, demostrando con ello que las características que este amparaba no eran consustanciales a la mujer, sino adquiridas como fruto de la imposición de un régimen androcéntrico. Lo fundamental, puntualizaba Laffitte, es que este nuevo trance histórico permita darle una nueva forma a su espíritu, aunque no señaló, por el momento, en qué habría de consistir esta nueva "forma"22.

Lo que Laffitte estaba describiendo implícitamente con estas palabras a finales de los ańos cincuenta era la llegada de nuevos paradigmas de mujer por influencia extranjera y mediante cauces entre los que sabemos que estuvieron el cine y la incipiente cultura del ocio, así como la peregrinación laica del turismo de playa. Pero lo que realmente le preocupaba a la escritora era que la mujer española copiara "como una nueva moda" la renovación de su papel en la sociedad y que "no evolucionara interiormente". Aunque la cuestión radicara en la situación de la mujer, la escritora la planteaba de un modo general así: “¿En qué nos mantenemos los españoles apegados a la tradición y en qué hemos aceptado renovarnos?”. Y se sentía tremendamente insegura al no poder contrastar sus impresiones con datos objetivos para dar respuesta a aquella pregunta: "Para un estudio de esta índole, por somero que sea como en este caso, habría que poseer un material de encuestas, test y estadísticas, documentos preciosísimos en los que desgraciadamente no abundamos" ${ }^{23}$.

En esta falta de información estuvo el germen de los nuevos proyectos que, individual y colectivamente, Laffitte realizaría a partir de 1960. Así, resulta verdaderamente significativo el hecho de que a finales de los cincuenta la escritora reclamara más material con el que trabajar, y que a principios de los sesenta se vea ya involucrada en dos tareas que requirieron y produjeron a la vez destacadas aportaciones en cuanto a datos y estudios se refiere. La

21 María Laffitte, La mujer como mito y como ser humano, Madrid, Taurus, p. 10.

22 Ibid., p. 22.

23 Ibid., p. 48-49. 
primera, un nuevo libro que se publicaría en 1964 titulado La mujer en España. Cien años de su historia; la segunda, la fundación en 1960 del Seminario de Estudios Sociológicos de la Mujer.

El primero de estos proyectos tuvo su origen en la propuesta por parte de la editorial Aguilar a Laffitte para que esta desarrollara el tema La vida social y Corte para la colección Panorama de un siglo. "Era tema, que si bien pudiera estar cerca de mi condición social, estaba, en cambio, lejos de mi personalidad como escritora", recordaba Laffitte ${ }^{24}$. Ante la renuncia, la editorial Aguilar dejó a la elección de la escritora el tema del volumen, y ella escogió La mujer en España. Cien años de su historia (1860-1969), problemática que decidió abordar a partir de una estructura tripartita (siglo XIX, 1900-1936 y 1939-1960) que dejaba fuera del estudio la Guerra Civil, según la escritora, por no haber encontrado un material que le permitiera abordar estos años con objetividad. En las últimas páginas de esta obra Laffitte añadió un capítulo titulado "Y ahora, algunos comentarios" donde pudo abordar las cuestiones específicas que, a principio de los ańos sesenta, continuaban ralentizando la emancipación de la mujer y la igualdad entre los dos géneros. La descripción de estos puntos hay que interpretarla como la elaboración de una prometedora agenda feminista, tal vez no tanto dirigida a los logros que aún quedaban por alcanzar, sino a los temas que debían ser analizados pormenorizadamente en un estudio sobre la situación de la mujer española. Así pues, en este capítulo final la escritora volvió a comparar la emancipación de la mujer española con la de la inglesa, que según Laffitte "buscó la solución a sus problemas a través de la política, de forma enérgica y hasta violenta", y con la francesa, que "da muestra de su capacidad privadamente dominando al hombre con una hábil mezcla de sensualidad e inteligencia" 25 . Con estas palabras Laffitte estaba tratando de corroborar la hipótesis que en aquel pequeño ensayo de La mujer como mito y como ser humano había propuesto: la de que la emancipación de la mujer española era una emancipación dada, no buscada, y que por tanto requeriría de un proceso de reajuste (y de su correspondiente estudio) entre los cambios efectivos que estaban llegando y la propia autopercepción de las mujeres respecto a su lugar en la sociedad.

En segundo lugar, la escritora afirmaba que para la mujer española no existía el sexo como satisfacción, ya que este quedaba anulado por la única función biológica de gestar, parir y lactar a sus hijos para la que la mujer se encontraba destinada. El placer sexual sólo parecía necesario, según denunciaba Laffitte, para el hombre, lo que daba lugar a una profunda desigualdad que desembocaba en relaciones desarticuladas y malogradas. Para ella "el amor surge del acorde armonioso entre lo sexual y lo espiritual. Actualmente -añadió- este esquema trazado está en plena evolución"26.

24 María Laffitte, Mi atardecer entre dos mundos, op. cit., p. 103-104.

25 Ibid., p. 377-379.

26 Ibid., p. 375. 
Por último, Laffitte abordó la insuficiencia de las trasformaciones sociales que habían llevado a la mujer a ascender en sus posiciones laborales e intelectuales. Aun reconociendo los progresos, insistió en que "la incorporación de la mujer al campo de las profesiones liberales es todavía un fenómeno sin asimilar", y puso de ejemplo el hecho de que fuera todavía "prácticamente inútil" que una mujer opositara a una cátedra universitaria. Esta situación planteaba para Laffitte muchas preguntas sobre la experiencia emancipadora del último siglo. "¿Es que la mujer española ha cubierto durante esta etapa todo su recorrido?", “¿cuándo tuvo, cuándo tendrá la mujer verdadera igualdad de oportunidades en relación al hombre?”27.

\section{El feminismo como proyecto colectivo}

Ciertamente, para principios de los sesenta, formulación de una agenda o de una hoja de ruta a seguir, la escritora decidió embarcarse en el proyecto que más satisfacción le reportaría, según ella misma aseguraba: la fundación del Seminario de Estudios Sociológicos de la Mujer, del que fue fundadora en 1960 y que dirigió hasta 1980. Junto con el cuestionamiento firme de los paradigmas de mujer franquista, este grupo de trabajo -que no fue legalizado hasta 1977- tuvo como objetivo averiguar cómo se estaba desarrollando el proceso de integración de la mujer en la modernización del país. Para ello, Laffitte había conseguido reunir, gracias a la colaboración de su amiga Lilí Álvarez, a un grupo de mujeres que cumplían el perfil que la escritora había establecido como necesario para integrar el grupo de trabajo: "que todas ellas fueran universitarias y que ejercieran una profesión a fin de que tuvieran una experiencia directa de lo que era, por entonces, el mundo del trabajo para la mujer" ${ }^{28}$. Compusieron el grupo Consuelo de la Gándara, Elena Catena, las hermanas Pura y María Salas Larrazábal, Concepción Borreguero Sierra, María Jiménez de Obispo del Valle y la ya citada Lilí Álvarez (Elia María González-Álvarez y López-Chicheri).

Aunque Laffitte definiera el SESM como un grupo con carácter "aconfesional y apolítico" 29 , en el primer proyecto que realizó el SESM se observaba todavía la preponderancia de unos valores católicos que consideraban a la religión como algo no solo compatible, sino necesario, para la correcta evolución de la mujer moderna. Esta primera labor consistió en la elaboración de un sondeo sociológico entre las mujeres madrileñas de diecisiete a treinta y cinco años de Madrid con el fin de aclarar la situación de la mujer española en el proceso de modernización. Las conclusiones, recogidas en Habla la mujer

27 Ibid., p. 348.

28 María Laffitte, Mi atardecer entre dos mundos, op. cit., p. 122.

29 María Laffitte, Mi atardecer entre dos mundos, op. cit., p. 123. 
(Cuadernos para el Diálogo), corroboraron para el SESM aquella carencia de un proceso gradual de evolución en las mujeres que Laffitte había apuntado en 1964. El estudio demostraba la pasividad de las jóvenes y su "inmovilismo y escasa evolución", entendiendo por ello "la falta de actitud interna ante los problemas sociales", y mostraba la falta de responsabilidad de las mujeres, probablemente a causa de la excesiva protección familiar. Además, el sondeo revelaba la escisión entre el mundo masculino y el femenino, es decir, la poca confianza, complicidad y entendimiento entre los dos géneros. Como señales positivas, el SESM destacaba la estima de los valores familiares, la "sana moralidad sexual", la religiosidad, la bondad y generosidad, y el optimismo ${ }^{30}$.

El siguiente estudio que realizó el SESM, Mujer y Aceleración histórica, fue publicado en la colección "Los Suplementos" de Cuadernos para el Diálogo en 1972. Laffitte señalaba a finales de esta década la intención explícita que tenía aquel estudio: era una "respuesta a la publicación del Libro Blanco para la reforma de la educación en España, con el que no estábamos totalmente de acuerdo en lo referente a la mujer"31. El Libro Blanco de la Educación había precedido a la Ley General de Educación de 1970 con la que se iniciaba la enseńanza mixta en España. La ambigüedad de sus planteamientos se puso de manifiesto desde su implantación, ya que esta norma no cuestionaba los valores tradicionales y el rol hogareño de las mujeres, sino que continuaba promoviendo para ellas los objetivos de ser esposas y amas de casa. Amparándose en el rol doméstico de las mujeres, nada aportó sobre la coeducación, con lo que perpetuó en esencia todo el entramado sexista, sin fracturar la visión tradicional ${ }^{32}$. Por su parte, Mujer y Aceleración Histórica incidió en esta continuidad de la discriminación por sexo en el ámbito de lo educativo: "El Estado, en este aspecto, no ha puesto mucho interés, hasta el presente, en modificar esta mentalidad y, en muchos casos, ha frenado ostensiblemente los intentos progresistas de incorporar plenamente a la mujer a la sociedad española"33. A la crítica la acompañaban propuestas concretas que defendían la urgente profesionalización del trabajo femenino y la necesidad de un régimen de coeducación ante la insuficiencia de una enseñanza mixta pero diferenciada por sexo.

30 Seminario de Estudios Sociológicos de la Mujer, Habla la mujer, resultado de un sondeo sobre la población actual. Madrid, Cuadernos para el Diálogo, 1967, p. 200.

31 María Laffitte, Mi atardecer entre dos mundos, op. cit., p. 124.

32 Concepción Borreguero, Elena Catena et al. (ed.), La mujer española: de la tradición a la modernidad. Madrid, Tecnos, 1968; Consuelo Flecha García, "Las mujeres en el Sistema Educativo Español”, en María Teresa Marín Eced y María del Mar Pozo Andrés (coords.), Las Mujeres en la construcción del mundo contemporáneo, Cuenca, Diputación Provincial; y Teresa González Pérez, "Mujeres, educación y democracia”, Revista de Educación, no 351, enero-abril 2010, p. 337-359.

33 Seminario de Estudios Sociológicos de la Mujer, Mujer y aceleración histórica. Madrid, Cuadernos para el Diálogo, 1971, p. 153-157. 
En 1977 el seminario publicaba Diagnosis sobre el amor y el sexo en la editorial Plaza \& Janés de Barcelona. El proyecto partió de la intención de investigar la afectividad de la pareja humana. "Limitamos nuestro estudio a los jóvenes de 18 a 21 años, porque entendemos que ya han pasado la adolescencia y todavía no están demasiado moldeados por la sociedad", explicaban las autoras ${ }^{34}$. En La mujer en España. Cien años de su historia, Laffitte había apuntado la diferencia entre las experiencias del hombre y de la mujer en cuanto al sexo, y para ella era uno de los temas que quedaban pendientes de análisis, por lo que parece bastante lógico que impulsara un estudio de estas características en el SESM. Las conclusiones extraídas por las autoras en esta obra, más renovadoras que las dos obras anteriores, advertían que se estaba asistiendo a mediados de la década de los setenta a una evolución de las relaciones interpersonales de los sexos, puesto que caracterizaba a la juventud "el deseo de superar los tabúes sexuales, movida por la convicción de que todo lo natural y espontáneo es bueno". Además de posicionarse a favor del divorcio, El SESM destacaba la "exigencia de una mayor autenticidad en el amor y en el matrimonio" y el hecho de que las relaciones sexuales prematrimoniales fueran cada vez más frecuentes y socialmente admitidas.

El proyecto frustrado del SESM fue la redacción de un libro sobre el movimiento feminista en España. Para su realización, el seminario invitó a sus reuniones a destacadas protagonistas del feminismo como Pilar Bellosino, Pilar Folquera o María Aurelia Capmany ${ }^{35}$. Moreno Seco ha visto en esta actitud del SESM una predisposición al diálogo con otros grupos feminista ${ }^{36}$, lo que se corrobora con su participación de los miembros del seminario en las Is Jornadas de Liberación de la Mujer organizadas por la Plataforma de Grupos Feministas en diciembre de $1975^{37}$ y respuesta, en parte, a las conmemoraciones oficialistas del que se había declarado como Año Internacional de la Mujer. Este evento significó la reunión de la mayoría de las organizaciones o grupos que durante los anteriores años de dictadura habían ido fraguando un pensamiento feminista, muy difuso y contradictorio en ocasiones, pero mantenido como auténtica resistencia al dogma sobre la mujer inculcado por el régimen.

34 Seminario de Estudios Sociológicos de la Mujer, Diagnosis sobre el amor y el sexo. Barcelona, Plaza \&Janés, 1977.

35 María Laffitte, Mi atardecer entre dos mundos, op. cit., p. 124.

36 Mónica Moreno Seco, y Alicia Mira Abad, "Maternidades y madres: en enfoque historiográfico", en Silvia Carporale-Bizzini, Discursos teóricos en torno a la(s) maternidad(es): una visión integradora, Barcelona, Entinema, 2005, p. 143.

37 Concepción Borreguero, Elena Catena et al. (ed.), La mujer española: de la tradición a la modernidad, op. cit., p. 32. 
En 1977, el SESM se pudo legalizar, y en sus estatutos reflejó una serie de objetivos que, para Moreno Seco, eran prueba también de su confluencia con el movimiento feminista ${ }^{38}$. El grupo se proponía, según sus objetivos, cambiar la mentalidad tradicional a través de una educación crítica y transformar las relaciones interpersonales de acuerdo con los presupuestos del feminismo. Además, se mostraba a favor de una ley que regulara el divorcio; de la información sobre anticonceptivos; de que las responsabilidades familiares fueran asumidas por el padre y la madre indistintamente; por la coeducación en todos los niveles de la enseñanza y por la aplicación rigurosa del principio de igual retribución por trabajo de valor equivalente. Estos fueron los principios que guiaron la actividad del SESM hasta su disolución "por acuerdo unánime y amistoso" en 1986, después del fallecimiento de Laffitte y de Consuelo de la Gándara ${ }^{39}$.

\section{Conclusiones}

En 1980 Laffitte dejaba de dirigir el SESM "por razones de edad y por haber abandonado el casco urbano para residir en sus cercanías" ${ }^{40}$, y en un capítulo de su autobiografía, titulado "Agonía del patriarcado", escrito contemporáneamente -a comienzos de la década de los ańos ochenta-, se identificaba ya con las aspiraciones del feminismo contemporáneo. La escritora, en una adscripción plena a la tradición del feminismo ilustrado, consideraba que este era un movimiento nacido con la Revolución Francesa, que había tomado cuerpo durante el siglo XIX, especialmente con las sufragistas inglesas, y que luego se había extendido hasta culminar, en los años sesenta, en Norteamérica. Por tanto, para principios de los ochenta el pensamiento de Laffitte había evolucionado, dejando atrás las nociones esencialistas de aquel "eterno femenino como instinto maternal" defendido en los años cuarenta y sustentado en las aportaciones de Concepción Arenal. Progresivamente, y en parte debido al trabajo colectivo del SESM, su visión del feminismo se había ampliado, y a la repercusión de las teorías de Simone de Beauvoir en los años cincuenta, se sumarían otras como las de feminismo católico o las del feminismo socialista, deudoras todas de la especial coyuntura reivindicativa del tardofranquismo y de la transición democrática.

Así, aunque la obra de esta escritora no se circunscribiera a su interés por el tema de la mujer, sino que englobara otros tantos ámbitos de conocimiento, la propia evolución que sufrieron sus concepciones feministas son un magní-

38 Mónica Moreno Seco, "Cristianas por el feminismo y la democracia”, op. cit., p. 143.

39 María Salas Larrazábal y Mercedes Comabellas, "Asociaciones de mujeres y movimientos feminista”, en Españolas en la transición. De excluidas a protagonistas (1973-1988), Madrid, Biblioteca Nueva, 1999, p. 29.

40 Concepción Borreguero y Elena Catena et al. (ed.), La mujer española: de la tradición a la modernidad, op. cit., p. 9. 
fico reflejo de los diferentes caminos que recorrió a lo largo de su trayectoria intelectual. El de María Laffitte fue siempre, y en todos los aspectos que abarcó, un pensamiento crítico que se abrió paulatinamente a los presupuestos liberales que con lentitud, pero también con eficacia, se fueron colando en la España de la dictadura y prepararon el terreno para la aparición de una cultura democrática durante y después del franquismo.

\author{
Begońa Barrera López \\ Universidad de Sevilla \\ begobarlop@gmail.com
}

\begin{abstract}
Resumen
La escritora María Laffitte desarrolló una amplia obra dedicada a cuestionar lo "femenino" desde nuevas perspectivas teóricas que escapasen a la normativa de género imperante durante el régimen franquista. Durante cuatro décadas, Laffitte desarrolló un pensamiento singular fruto de la convergencia entre importantes corrientes filosóficas de su tiempo y su propia experiencia vital.
\end{abstract}

\title{
Palabras claves
}

María Laffitte, femenino, mujer, género, franquismo.

\section{Résumé}

L'œuvre prolifique de l'écrivain espagnole Marie Laffitte reflète le besoin de remettre en question le "féminin " à partir de positions éloignées de la normalisation du genre tel qu’il avait été conçu sous le régime franquiste. Et pendant quatre décennies, elle a élaboré une pensée singulière située à la croisée de ses positions personnelles et des influences des grands théoriciens de son temps.

\section{Mots Clés}

María Laffitte, féminin, femme, genre, franquisme.

\begin{abstract}
Maria Lafitte developed a prolific career as a writer, which reflected her interest in rethinking the idea of the feminine, drawing from her independent position to the social norms stipulated by the Franco regime. Over forty years she converged both personal contributions and influences with the dominant theoretical thinking of the time.
\end{abstract}

\section{keywords}

María Laffitte, female, woman, gender, francoism. 